\title{
Combination of EGFR and COX-2 inhibitors in breast cancer patient
}

\author{
Viroj Wiwanitkit
}

Received: 22 January 2012 / Accepted: 31 January 2012 /Published online: 24 February 2012

(C) International Society of Oncology and BioMarkers (ISOBM) 2012

\begin{abstract}
Ther combination of EGFR and COX-2 inhibitors in breast cancer patient treatment is a topic of interest. In this work, the author discusses on the synergistic effect of concomitant usage of the two drugs and also further notes on the myth in using this combination.
\end{abstract}

\section{Keywords EGFR $\cdot$ COX-2 $\cdot$ Inhibitor $\cdot$ Breast $\cdot$ Cancer}

Sir, the recent publication on "combination of EGFR and COX-2 inhibitors in breast cancer patient [1]" is very interesting. Wang et al. concluded that "nimotuzumab and celecoxib exert synergistic antiproliferation effects in breast cancer, which partly correlates with ER status [1]." Here, I would like to share experience on this topic. The case is a case with ER positive stage 4 breast cancer and failed from conventional treatment strategies of chemotherapy, radiation, and antiestrogen therapy. The case was consulted to get nimotuzumab. Also, the patient had the problem of back pain and simultaneously treated by celecoxib. Of interest, the significant reduction of the erythrocyte sedimentation rate and C-reactive protein in this case could be observed within 1 month (three times reduction to normal level) after starting of nimotuzumab. The reduction in this case might be due to the nimotuzumab or the synergistic effect of the combination as report by Wang et al. [1]. Since this is not a trial, there is no control group and the use of combination regimen in this case is by chance. It cannot be said that there is an additional reduction of the biomarkers due to adding celecoxib to nimotuzumab. Further investigation on this topic is required. Nevertheless, focusing on the action of each drug, the nimotuzumab has direct effect of epidermal growth factor [2] and the suppression of EGF due to celecoxib [3,4] is reported. Hence, it is no doubt that the synergistic effect might be possible. Nevertheless, the myths are still left. The questions include (a) the pattern on possible synergistic action, (b) the limitation of synergistic effect, and (c) any possible side effect.

\section{References}

1. Wang YX, Gao JX, Wang XY, Zhang L, Liu CM (2012) Antiproliferative effects of selective cyclooxygenase- 2 inhibitor modulated by nimotuzumab in estrogen-dependent breast cancer cells. Tumour Biol

2. Zhu Z. Targeted cancer therapies based on antibodies directed against epidermal growth factor receptor: status and perspectives. Acta Pharmacol Sin. 2007;28(9):1476-93.

3. Bocca C, Bozzo F, Cannito S, Parola M, Miglietta A. Celecoxib inactivates epithelial-mesenchymal transition stimulated by hypoxia and/or epidermal growth factor in colon cancer cells. Mol Carcinog. 2011. doi: $10.1002 / \mathrm{mc} .20846$.

4. Ghosh N, Chaki R, Mandal V, Mandal SC. COX-2 as a target for cancer chemotherapy. Pharmacol Rep. 2010;62(2):233-44.

\section{Wiwanitkit $(\bowtie)$}

Wiwanitkit House,

Bangkhae,

Bangkok, Thailand

e-mail: wviroj@yahoo.com 